\section{Klinische Untersuchung der Halte- und Bewegungsorgane}

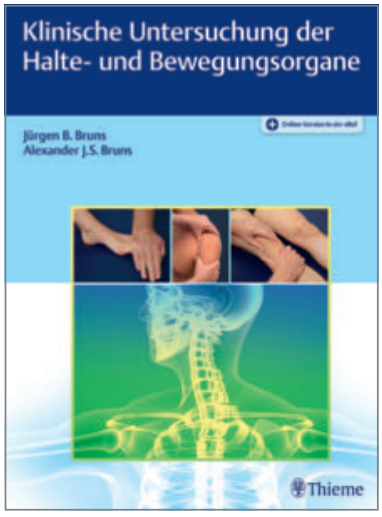

Bruns J. B., Bruns A. J. S. Stuttgart: Georg Thieme Verlag 2019; 256S, 602 Abb, 107,99€. ISBN 9783132050617

In den letzten Jahrzehnten hat die bildgebende Diagnostik insbesondere durch Sonografie, Computertomografie und Kernspintomografie einen sehr hohen Stellenwert erlangt. Dies hat leider dazu geführt, dass in der klinischen Ausbildung die rudimentäre klinische Untersuchung wohl noch gelehrt wird, spezielle Tests hingegen, welche Rückschlüsse auf die Anatomie und deren Pathologien zulassen, zunehmend in Vergessenheit geraten sind. Zudem wurden zahlreiche weitere klinische Tests im Laufe der Zeit entwickelt und verfeinert, welche zum Teil nur dem Experten für die jeweili- ge Körperregion bzw. das jeweilige Gelenk bekannt sind. Die klinische Untersuchung lässt sich relativ schlecht textlich erklären. Aus diesem Grund ist das vorliegende Buch besonders positiv hervorzuheben, da durch zahlreiche klinische Bilder die Tests so anschaulich dargestellt und beschrieben werden, dass diese auch vom ungeübten Untersuchenden durchgeführt werden können. Dennoch ist es natürlich selbstverständlich notwendig, die klinische Untersuchung in der täglichen Praxis zu üben und die Anwendung durch eine/n erfahrene/n Untersucher/in überprüfen zu lassen, um letztendlich Sicherheit zu gewinnen.

Das Werk ist systematisch nach anatomischen Regionen geordnet. Alle Untersuchungstechniken werden an klinischen Bildern, ergänzt durch zahlreiche Schemazeichnungen, demonstriert. Besonders hervorzuheben sind die Textkästen, die auf mögliche Fehler ebenso hinweisen wie auf Pathologien nach denen explizit gesucht werden muss, wie z. B. dem Kompartmentsyndrom des Unterschenkels bei Verletzung der unteren Extremität. Neben den klinischen Tests der Gelenke und der Wirbelsäule wird auch die neurologische Untersuchung dezidiert beschrieben. So werden die Dermatome der verschiedenen Körperregionen in Schemazeichnungen farblich gut voneinander abgrenzbar dargestellt. Sehr viele Tests sind den Untersuchungstechniken der manuellen Me-

Nach der kurzgefassten Handchirurgie, welche eine langjährige Tradition hat, und der kurgefassten Fußchirurgie erscheint nun die kurzgefasste Schulterchirurgie im Thieme Verlag. Nach einer einleitenden Darstellung der Schulteranatomie und der klinischen Tests sowie der apparativen Diagnostik und hier insbesondere der Kernspintomografie und der Schultersonografie, wird die Lagerung zur Operation ausführlich dargestellt.

Zu den Krankheitsbildern des Schultergürtels und des Glenohumeralgelenkes werden sowohl die konservativen als auch die operativen Verfahren prägnant dargestellt. Das Buch ist sehr reich an farbigen klinischen Bildern, aber auch an Abbildungen der bildgebenden Diagnostik von ausgezeichneter Schärfe. Ergänzt werden diese durch sehr dizin entnommen und helfen dem Untersuchenden sehr spezifisch die Schmerzursache bzw. die Bewegungsstörung auch dort zu erkennen, wo viele Gelenke nah beieinander liegen, wie z. B. in der Handwurzel, der Fußwurzel oder an der Wirbelsäule.

Zusammenfassend bietet das Werk einen sehr guten Überblick über die Untersuchungstechniken des Bewegungsapparates und ist somit schon Studierenden im praktischen Jahr aber auch jungen AssistentInnen besonders zu empfehlen. In Zeiten zunehmender Spezialisierung der Ärzteschaft ist es auch erfahrenen KollegInnen zur Rekapitulation in Körperregionen, welche sie nicht so häufig untersuchen, eine willkommene Hilfe. Nicht nur OrthopädInnen und Unfallchirurglnnen, sondern auch internistischen RheumatologInnen und HausärztInnen ist dieses Buch sehr zu empfehlen, um unbekannte, in Arztbriefen beschriebene Tests in Wort und Bild sehr schnell nachzuvollziehen.

Prof. Dr. Ralph Gaulke, Hannover

ansprechende und übersichtliche Schemazeichnungen.

Die „Kurzgefasste Schulterchirurgie“ ist somit allen OrthopädInnen und Unfallchirurglnnen zu empfehlen, welche sich zum einen einen Überblick über die Subdisziplin der Schulterchirurgie verschaffen wollen und zum anderen ein Werk suchen, in dem sie ihr Wissen kurz und knapp rekapitulieren können.

Prof. Dr. Ralph Gaulke, Hannover
Frank J., Meyer R. Stuttgart, New York: Georg Thieme Verlag 2020. 296S., 542 Abb., 157,99€. ISBN 9783131983213 


\section{Manual of Fracture Management - Wrist}

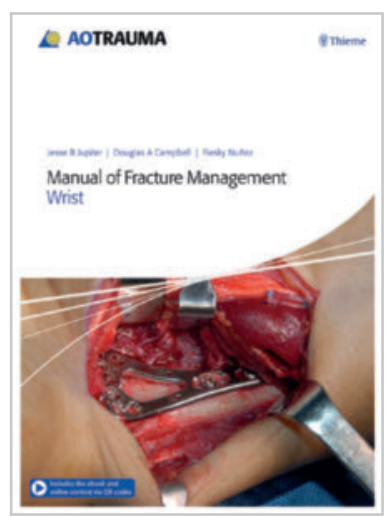

Jupiter J. B., Campbell D. A., Nunez F. A. Stuttgart, New York: Georg Thieme Verlag 2019; 524S., 2000 Abb., 174,99€. ISBN 9783132428416

Das englischsprachige Werk der AO Trauma zur Frakturversorgung am Handgelenk stellt sehr ausführlich und reich bebildert die AO-Techniken zur Versorgung von Frakturen des distalen Radius sowie der Handwurzelknochen dar. Da das Buch von der AO
Trauma herausgegeben wird, werden ausschließlich Operationstechniken mit Implantaten der AO dargestellt. Die Techniken sind die gleichen, welche seit Jahren in den Kursen der AO vermittelt werden.

Die verschiedenen Frakturtypen werden in ihrer Differenzialtherapie in Bezug auf die Repositions- und Fixationstechniken ausführlich dargestellt. Die Philosophien und Indikation für die verschiedenen Plattendesigns werden ausführlich erläutert. Da das Buch insgesamt von den Bildern lebt und wenig Text enthält, ist es auch für Leserinnen und Leser, die lediglich über englische Grundkenntnisse verfügen, sehr gut verständlich. Auch minimalinvasive Plattenosteosynthesen bei distalen Radiusfrakturen durch sogenannte Brückenplatten, welche funktionell einen Fixateur interne darstellen, zwischen dem Radius und dem Mittelhandknochen werden ausführlich dargestellt. Auf mögliche technische Fehler wird ausführlich hingewiesen. Aufgrund dessen, dass die meisten Autorinnen und Autoren aus Amerika kommen, sind die

säule ebenso neigt wie zu Frakturen nach Bagatellverletzungen.

Bevor das Buch sich mit den Therapien in der Alterstraumatologie an der Wirbelsäule auseinandersetzt, werden zunächst die Besonderheiten des älteren Menschen sowie altersbedingte Veränderungen an der Wirbelsäule dargelegt. Die Diagnostik und die Therapie der Osteoporose beim alternden Wirbelsäulenpatienten werden ausführlich behandelt. Noch wichtiger als die Operationstechnik erscheint die Indikationsstellung für eine konservative oder operative Therapie im Lichte der Nebenerkrankungen und des Aktivitätsanspruches des Patienten. Auch die optimale präoperative Vorbereitung, welche sehr wichtig ist, damit die Patienten den Eingriff gut überstehen und sich hinterher schnell von diesem erholen, wird systematisch behandelt.

Die Altersmedizin rückt zunehmend in de Blickpunkt, da ältere Menschen länger aktiv bleiben und sich dadurch die Gefahr von Verletzungen erhöht. Außerdem werden die Menschen im Durchschnitt immer älter, wodurch praktisch Jede/r im Laufe seines Lebens eine Osteoporose entwickelt und somit zu spontanen Frakturen der Wirbel-
Zugänge über dem Handgelenk meistens gerade in Längsrichtung ausgerichtet und nicht geschwungen, wie sie im deutschsprachigen Raum in handchirurgischen Kreisen üblich sind, um einer postoperativen Bewegungseinschränkung durch eine Kontraktur der Narbe entgegenzuwirken. Auch Korrekturoperationen nach fehlverheilten Frakturen werden ausführlich dargestellt. Entsprechende Videos sind online verfügbar.

Zusammenfassend beinhaltet das Buch eine ausführliche Anleitung für all diejenigen, welche sich mit der operativen Therapie frischer Handgelenkfrakturen sowie der Korrektur fehlverheilter Frakturen beschäftigen. Auch für Leserinnen und Leser, die Implantate anderer Hersteller bevorzugen, hält das Buch zahlreiche Tipps und Tricks bereit, die die Frakturversorgung sehr erleichtern.

Prof. Dr. Ralph Gaulke, Hannover

und ohne Myelopathie sowie der lumbalen Stenose und degenerativen Halswirbelsäulenveränderungen gewidmet. Auch die nichtchirurgische Schmerztherapie wird mit ihren Fallstricken beschrieben.

Den Abschluss bilden 3 Kapitel zur Augmentation bei Insuffizienzfrakturen, der minimalinvasiven Chirurgie im Alter und Techniken der Instrumentierung. Die Texte sind klar verständlich in kurze Abschnitte gegliedert, sodass diese auch für nicht englische Muttersprachler gut verständlich dargelegt sind. Erfreulich ist der konventionelle Aufbau mit Literaturangaben am Ende jeden Kapitels, was die mühsame Suche am Ende des Gesamtwerkes erspart.

Insgesamt ist dieses Buch nicht nur für Wirbelsäulenchirurglnnen, sondern für alle OrthopädInnen und UnfallchirurgInnen sehr zu empfehlen, da die Anzahl der traumatischen und degenerativen Veränderungen der Wirbelsäule aus obengenannten Gründen weiter zunehmen wird.

Prof. Dr. Ralph Gaulke, Hannover 